\title{
Suture Anchor Construct for an Exogenous Ligament Repair for Subtalar Joint Instability-A Novel Technique
}

\author{
Paul J Carroll* and Jonathan J Sharpe
}

Division of Podiatric Surgery, Orthopedic Associates of Lake County, USA

*Corresponding author: Paul Carroll, Fellow, Northern Ohio Foot and Ankle Reconstruction Fellowship, Division of Podiatric Surgery, Orthopedic Associates of Lake County, Concord OH, Lake Health, 7551 Fredle Dr. Concord, OH 44077, USA, Tel: 440-350-9595, Fax: 440-357-1905

\begin{abstract}
Subtalar joint ligament sprains are often missed in the setting of chronic inversion ankle injuries. This can lead to pain and instability of the subtalar joint. In many cases, without proper identification, the patient may eventually undergo an unnecessary subtalar joint fusion rather than a ligament reconstruction. Achilles, peroneus brevis, and gracilis tendon grafts have been described to repair subtalar ligament instability in the literature. To our knowledge, the use of exogenous ligament repair technique using nonabsorbable suture made of Ultra High Molecular Weight Polyethylene (UHMWPE) and suture anchors has not been described for subtalar joint stabilization. Here we present a case of 17-year-old female who underwent subtalar joint stabilization with a suture anchor construct for bilateral chronic subtalar joint instability.
\end{abstract}

\section{Keywords}

Ligament reconstruction, Subtalar instability, Talocalcaneal interosseous ligament, Cervical ligament, Ultra-high molecular weight polyethylene suture anchor construct

\section{Introduction}

In an acute inversion ankle injury, the most commonly injured structure is the anterior talofibular ligament (ATFL). Approximately, $80 \%$ to $85 \%$ of these injuries go onto heal with conservative treatment with rehabilitation [1]. However, $74 \%$ of these injuries may develop some degree of chronic joint instability [1,2]. Up to $75 \%$ to $80 \%$ of recurrent injuries may also present with chronic subtalar joint instability [1,2]. Many experts argue the calcaneofibular ligament (CFL) is the primary stabilizer of the subtalar joint [3]. Others suggest the primary stabilizers of the subtalar joint are the interosseous talocalcaneal ligament (ITCL) and cervical ligament. This has led to the debate as to which injured ligament is the cause of subtalar instability. Ultimately, the function of the ITCL is poorly understood. It has been hypothesized that the ligament limits eversion and maintains apposition of the talus and calcaneus in all positions [4]. In several cadaveric studies, sectioning the talocalcaneal interosseous ligament increased supination. The cervical ligament has been identified to limit supination and limit excessive motion of the subtalar joint [5]. There is debate on which of these two ligaments is the primary and secondary stabilizer of the subtalar joint [3]. If subtalar joint instability is correctly identified it can be treated with a joint sparing reconstructive ligament procedure. Currently, it has been reported in the literature that the subtalar joint has been stabilized with achilles, peroneus brevis and gracilis tendon allografts and autografts. This is a unique case study of a patient who underwent a subtalar joint ligament stabilization with an exogenous ligament repair technique using non-absorbable suture made of UltraHigh Molecular Weight Polyethylene (UHMWPE) and suture anchors. This specific technique is frequently employed for lateral ankle stabilization surgery, but to our knowledge has not yet been described for subtalar joint stabilization.

\section{Case Report}

A 17-year-old female with a one-year history of right foot and ankle pain after being hit in the foot with a softball and subsequent spraining injury to the ankle was treated by the author (JJS). Her only know past 


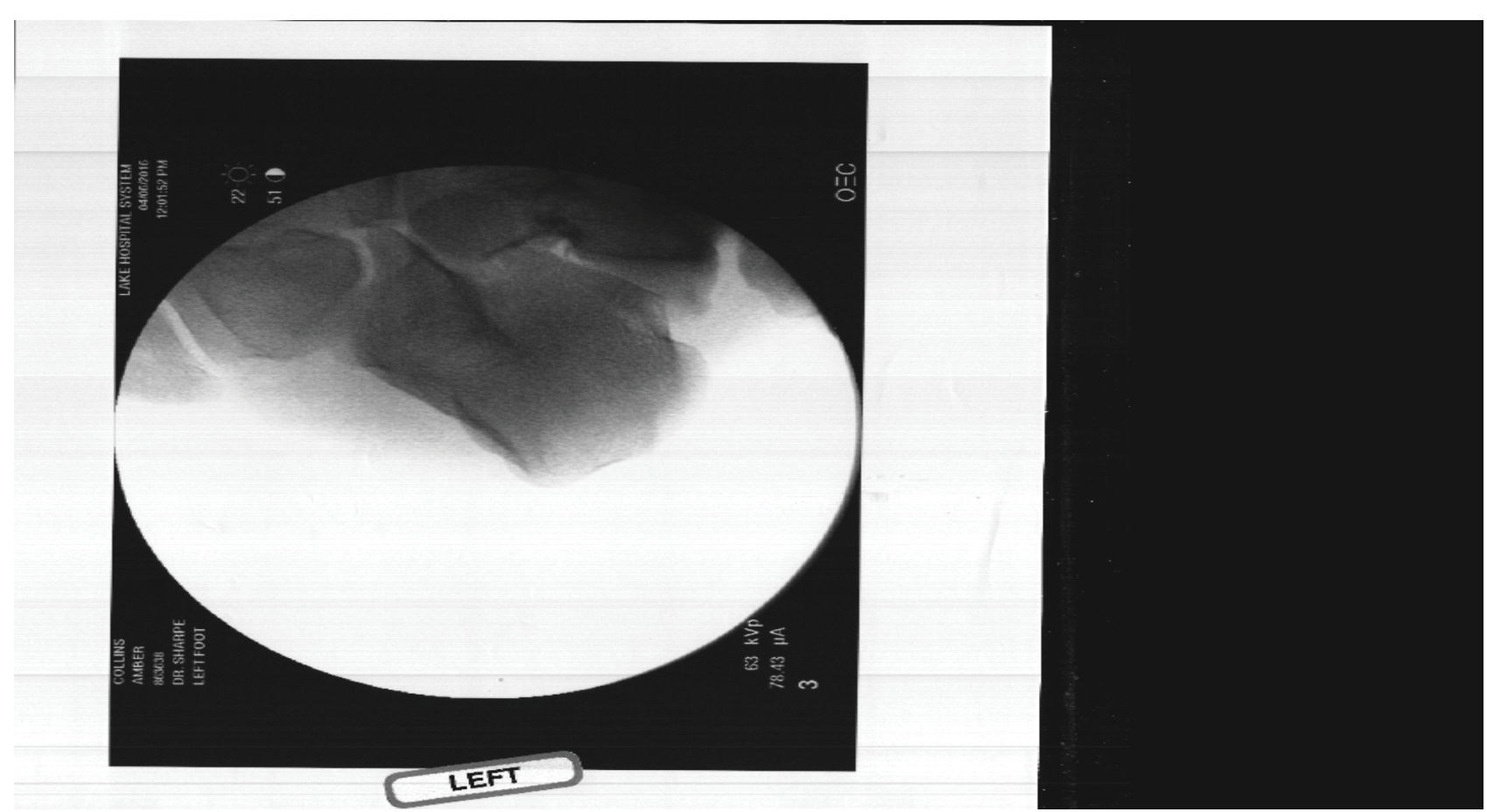

Figure 1: A) Subtalar joint prior to stress; B) Stressing of the subtalar on Broden's view, significant translation of the subtalar joint noted.

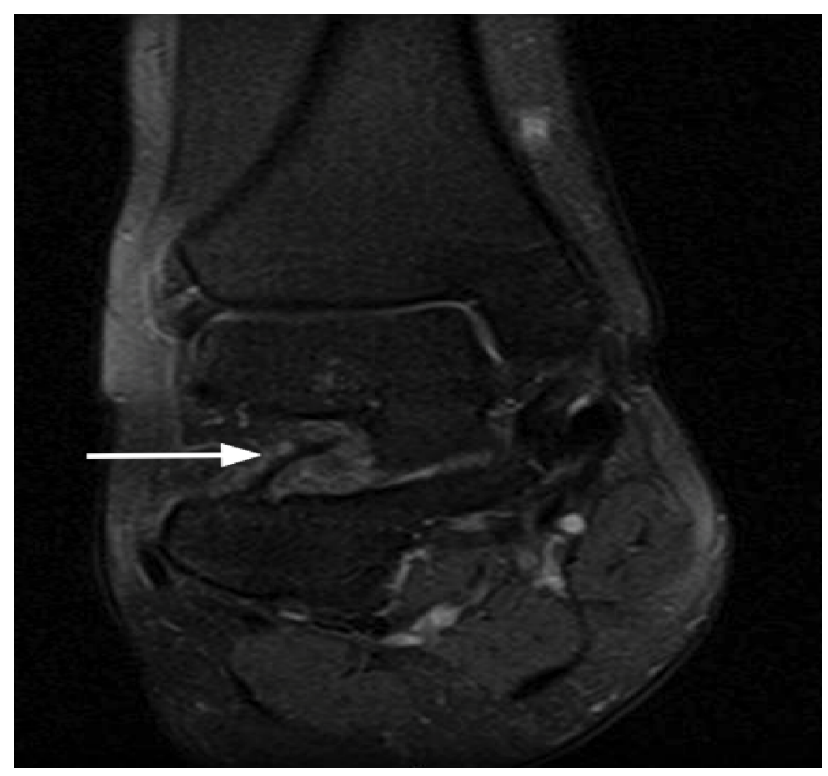

A

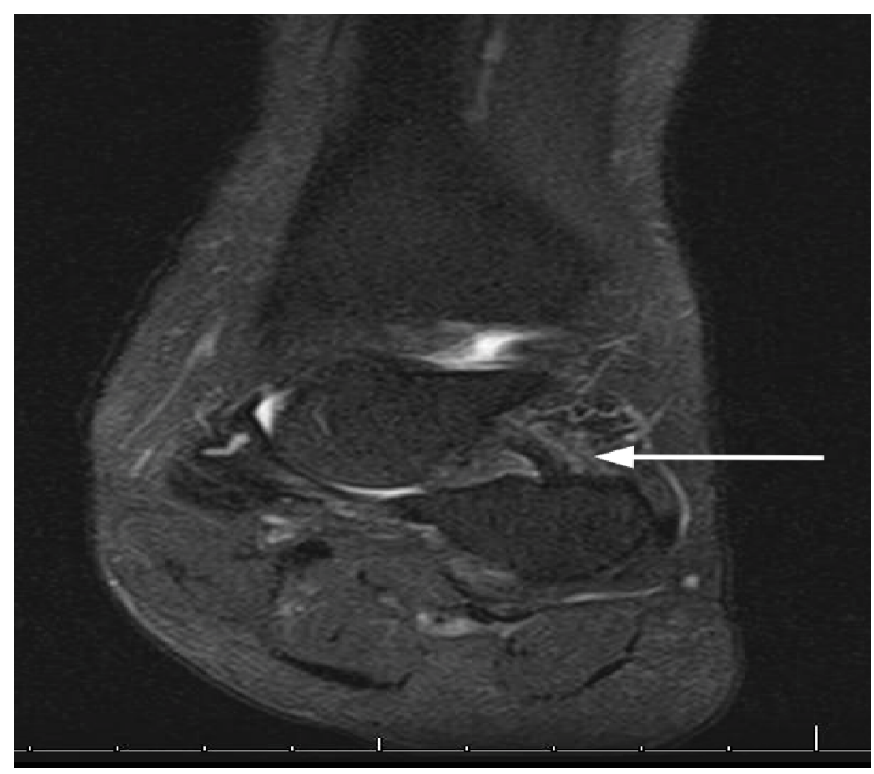

B

Figure 2: A.B) MRI contrasting chronically thickened cervical ligament on the right compared to healthy cervical ligament on the left.

medical history is Pott's disease. Her only prior treatment for this injury consisted of ankle compression wrapping. X-rays at the time of the injury were negative for fracture, arthrosis, or dislocation. Upon physical examination, there was pain on stressing of the right subtalar joint, inversion and eversion of the subtalar joint and palpation of the sinus tarsi. Stress radiographs of the ankle showed a negative anterior drawer and a negative talar tilt. However, stress of subtalar joint on Broden's view noted increased translation of the joint (Figure 1). She was prescribed custom orthotics and started a regiment of physical therapy. After 17 sessions of physical therapy, she noted minimal improvement. An MRI showed chronic, thickening of the cervical ligament (Figure 2). The MRI also demonstrated normal morphology to the calcaneofibular (CFL) and anterior talofibular (ATFL) ligaments. The patient and her family at this point opted for surgical exploration and stabilization of the subtalar joint.

The patient was placed supine on the operating room table with an ipsilateral hip bump. A well-padded mid-thigh tourniquet was placed. Once under anesthe- 


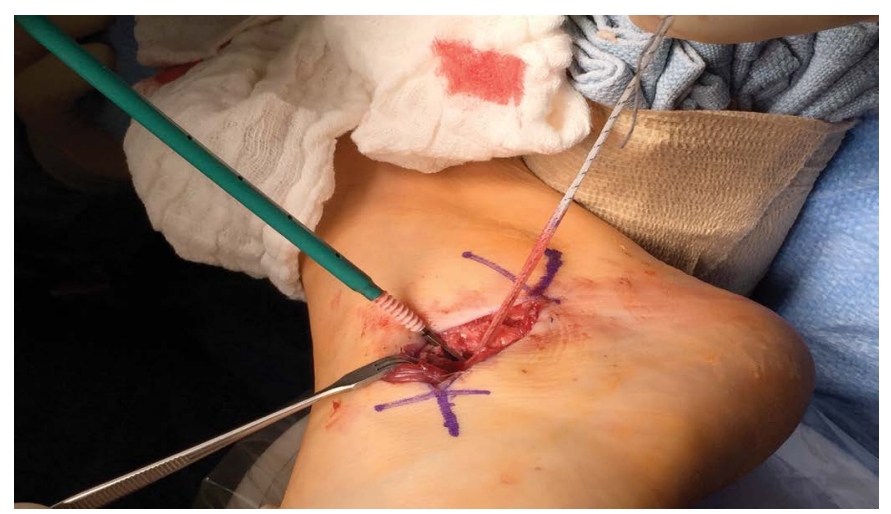

A

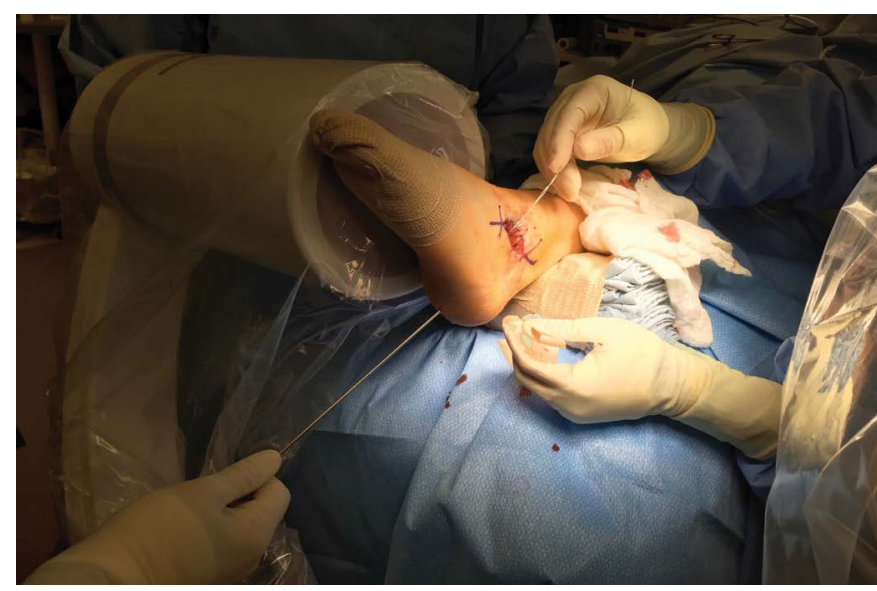

C

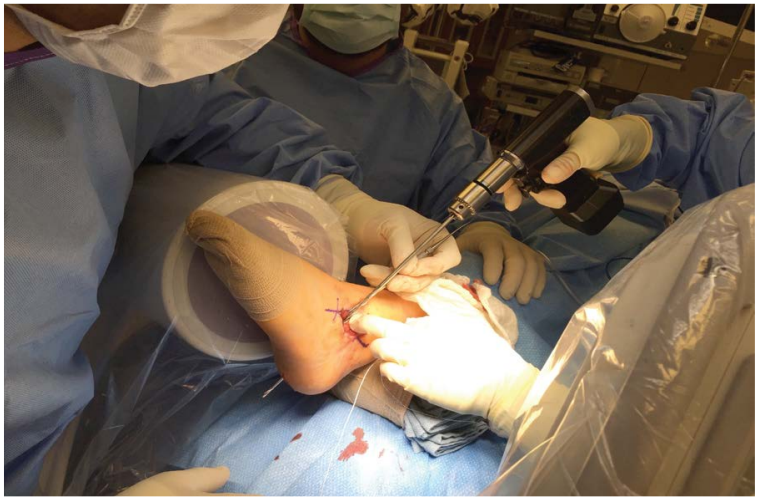

$\mathrm{B}$

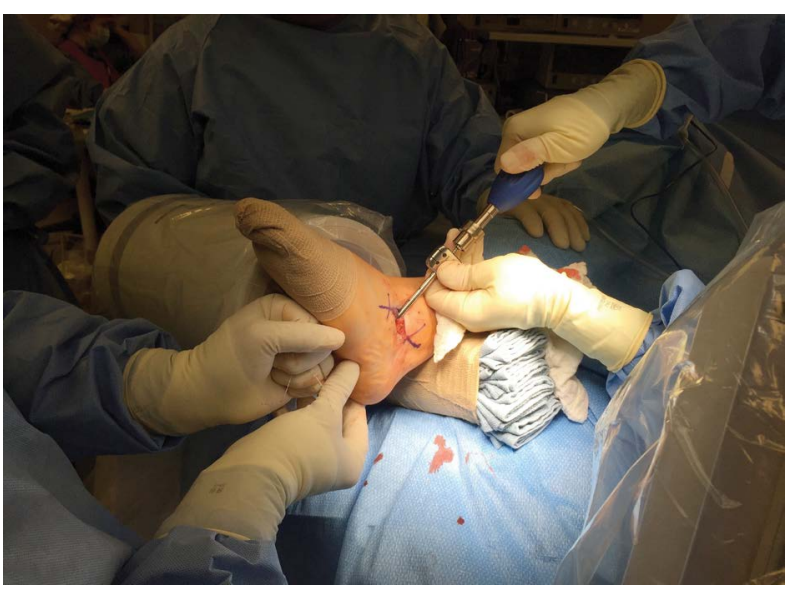

D

Figure 3: A-D) Reconstruction of the interosseous talocalcaneal ligament.

sia, the patient's subtalar joint was stressed under fluoroscopy showing clear instability. The lateral collateral ligaments were re-stressed under anesthesia and again did not show any evidence of mechanical instability. A four-centimeter lazy $S$ incision was made over the sinus tarsi. Blunt dissection was carried down to the sinus tarsi of the subtalar joint. Inspection of the subtalar joint ligaments revealed extensive scarring and laxity of the 7 cervical ligament and a normal morphology to the ITCL. No joint arthrosis was appreciated clinically. The cervical ligament was then stabilized with an $4.75 \mathrm{~mm}$ bioswivel lock anchor (Arthrex, Naples FL). A punch tap was performed just posterior to the anterior process of the calcaneus in the floor of the sinus tarsi. The associated UHMWPE fibertape suture was then looped through the cervical ligament and inferior extensor retinaculum and subsequently secured in the roof of the sinus tarsi on the inferior talar neck. A second $4.75 \mathrm{~mm}$ bio-swivel lock anchor was utilized for this. Following repair, the joint was irrigated with normal saline and a layer closure was performed (Figure 3). Post-operatively, the patient remained non-weight bearing for seven weeks with crutches. She was immobilized in a removable cast boot with immediate mobilization and range of motion encouraged on the operative foot. Following completion of physical therapy, the patient was able return to her normal activities in orthotics with minimal pain reported and no instability. Return to normal activity was permitted after four months of recovery postoperatively.

Six months following stabilization of her right subtalar joint, the patient returns to clinic with a reported left ankle sprain. The patient fell and heard a pop inside her ankle. Following a period of protected weight bearing and formal physical therapy, the patient still reported pain and instability in her left ankle. An MRI demonstrated chronic thickening of the cervical ligament of the left subtalar joint. Again, no ankle ligament instability or abnormalities were detected. Stress views indicated no mechanical failure of the ATFL or CFL. The patient continued conservative treatment of orthotics and physical therapy without relief. One year after the injury and no improvement of symptoms, the patient opted for surgical reconstruction of the left subtalar joint ligaments. The patient was positioned on the operating room table in a similar position as the previous foot surgery. There was radiographic evidence of subtalar joint instability noted on fluoroscopic stress views in the operative suite and again, negative ankle stress views. A curvilinear incision was made over the sinus tarsi. Blunt dissection was carried down to the subtalar joint. Both the interosseous talocalcaneal and cervical ligaments were noted to be scarred and thickened with laxity on stressing. An Arthrex $3.75 \mathrm{~mm}$ bio-composite tenodesis screw with accompanying fibertape (Arthrex, Naples FL) was inserted 
in the inferior portion of the neck of the talus on the roof of the sinus tarsi per manufacture recommended technique. Next, an osseous tunnel was drilled drill through the calcaneal floor of the sinus tarsi just posterior to the anterior process of the calcaneus from dorsal to plantar. The $4.75 \mathrm{~mm}$ punch tap was then utilized in preparation for the bio-composite screw. The fibertape was fed through the tunnel with a keith needle and pulled through the plantar skin on the non-weightbearing portion of the foot just distal to the heel. The suture was then tensioned and secured with a 4.75 bio-composite interference screw. The joint was irrigated. The inferior extensor retinaculum was imbricated into the repair. A layered closure was performed. The patient remained non-weight bearing for seven weeks and transitioned to protected weight bearing in a boot. During her recovery period, the patient fell with the foot unprotected and fractured the anterior process of her calcaneus on her right foot. This was identified on MRI examination. The fracture did not occur at or around the tenodesis screw but may have been associated with weakening secondary to the surgical reconstruction. This limited her immediate rehabilitation of her ligament repair on her left foot. Physical evaluation noted there was no laxity in the subtalar joint following trauma. It was clinically determined the repair remained intact. The new traumatic fracture was treated with immobilization for an additional eight weeks in a cast boot with weightbearing to tolerance. At 18 months after surgery the patient reports her joint is stable, but still painful. At that point she had not returned to full athletic activity.

\section{Discussion}

This case study discusses our surgical treatment of subtalar joint instability using an exogenous suture anchor construct. Our surgical technique and outcomes are presented. Following the repair of the cervical ligament for the stabilization of the subtalar joint of the right foot the patient was able to return to her normal activities without limitations. However, after her subtalar joint stabilization of her left foot, she continues to have pain which limits her activity. Similar repairs have been discussed in the literature. Jung, et al. reported great results in 20 ankles that underwent subtalar instability reconstruction with a semitendinosus allograft [6]. In their technique, the graft was placed between the ITCL and cervical, through the distal fibula and inserted in the calcaneus. The average pre-operative AOFAS score was 66 and improved 89 postoperatively. Liu, et al. reported excellent results in 1 patient who underwent arthroscopic repair of the ITCL using gracilis tendon autograft. The AOFAS score improved from 55 pre-operatively to 100 post-operatively [7]. Many other procedures have described in the treatment of subtalar instability. Following any repair of the subtalar joint ligaments, aggressive physical therapy is recommended to regain mobility and decrease pain following the procedure. If subtalar joint instability goes undiagnosed, this can lead to additional sprains as a result of altered subtalar joint mechanics [3]. Exogenous ligament repair of the ITCL and cervical ligament spares harvesting tendons for autograft, which can jeopardize their function in various capacities in the body or lead to unnecessary comorbidity. The use of UHMWPE suture for the repair of ITCL and cervical ligaments 10 was successful in re-stabilizing the subtalar joint in both feet in this case, however this patient continued to have chronic pain in one of the surgically repaired feet. More studies are needed before this construct can be recommended. Additionally, long term follow up is needed to evaluate the longevity of the repair with the suture anchor construct. This joint sparing procedure is an alternative to arthrodesis and tendon autograft.

\section{References}

1. Choisne J, Hoch M, Bawab S, Alexander I, Ringleb S (2013) The effects of a semi-rigid ankle brace on a simulated isolated subtalar joint instability. J Orthop Res 31: 1869-1875.

2. Choisne J, Hoch M, Alexander I, Ringleb (2017) Effect of direct ligament repair and tenodesis reconstruction on simulated subtalar joint instability. Foot Ankle Int 38: 324-330.

3. Keefe D, Haddad S (2002) Subtalar instability Etiology, diagnosis, and management. Foot Ankle Clin 7: 577-609.

4. Tochigi $\mathrm{Y}$, Amendola A, Rudert M, Baer T, Brown T, et al. (2004) The role of the interosseous talocalcaneal ligament in subtalar joint stability. Foot Ankle Int 25: 588-596.

5. Knudson G, Kitaoka H, Lu C, Luo Z, An K (1997) Subtalar joint stability. Talocalcaneal interosseous ligament function studied in cadaver specimens. Acta Orthop Scand 5: 442446.

6. Jung H, Park J, Shin M, Lee S, Eom J, et al. (2015) Outcome of subtalar instability reconstruction using the semitendinosus allograft tendon and biotenodesis screws. Knee Surg Sports Traumatol Arthrosc 23: 2376-2383.

7. Lui C, Jiao C, Hu Y, Guo Q, Wand C, et al. (2011) Interosseous talocalcaneal ligament reconstruction with hamstring autograft subtalar arthroscopy: Case Report. Foot Ankle Int 32: 1089-1094. 